\title{
Direct Detection of Drug-Resistant Hepatitis B Virus in Serum Using a Dendron-Modified Microarray
}

\author{
Doo Hyun Kim ${ }^{1}$, Hong Seok Kang ${ }^{1}$, Seong-Suk Hur ${ }^{1}$, Seobo Sim ${ }^{2}$, Sung Hyun Ahn ${ }^{1}$, Yong Kwang Park ${ }^{1}$, Eun-Sook Park ${ }^{1}$, Ah \\ Ram Lee ${ }^{1}$, Soree Park ${ }^{1}$, So Young Kwon ${ }^{3}$, Jeong-Hoon Lee ${ }^{4}$, and Kyun-Hwan Kim ${ }^{1,5,6}$ \\ ${ }^{1}$ Department of Pharmacology, Center for Cancer Research and Diagnostic Medicine, IBST, and Departments of ${ }^{2}$ Environmental and Tropical \\ Medicine and ${ }^{3}$ Internal Medicine, Konkuk University School of Medicine, ${ }^{4}$ Department of Internal Medicine and Liver Research Institute, Seoul \\ National University Hospital, Seoul National University College of Medicine, ${ }^{5} \mathrm{KU}$ Open Innovation Center and ${ }^{6}$ Research Institute of Medical \\ Sciences, Konkuk University, Seoul, Korea
}

Background/Aims: Direct sequencing is the gold standard for the detection of drug-resistance mutations in hepatitis $B$ virus (HBV); however, this procedure is time-consuming, laborintensive, and difficult to adapt to high-throughput screening. In this study, we aimed to develop a dendron-modified DNA microarray for the detection of genotypic resistance mutations and evaluate its efficiency. Methods: The specificity, sensitivity, and selectivity of dendron-modified slides for the detection of representative drug-resistance mutations were evaluated and compared to those of conventional slides. The diagnostic accuracy was validated using sera obtained from 13 patients who developed viral breakthrough during lamivudine, adefovir, or entecavir therapy and compared with the accuracy of restriction fragment mass polymorphism and direct sequencing data. Results: The dendron-modified slides significantly outperformed the conventional microarray slides and were able to detect HBV DNA at a very low level (1 copy/ $\mu \mathrm{L}$ ). Notably, HBV mutants could be detected in the chronic hepatitis B patient sera without virus purification. The validation of our data revealed that this technique is fully compatible with sequencing data of drug-resistant HBV. Conclusions: We developed a novel diagnostic technique for the simultaneous detection of several drug-resistance mutations using a dendron-modified DNA microarray. This technique can be directly applied to sera from chronic hepatitis $B$ patients who show resistance to several nucleos(t)ide analogues. (Gut Liver 2018;12:331-341)

Key Words: Hepatitis B virus; DNA microarray; Dendron; Drug resistance

\section{INTRODUCTION}

Hepatitis B virus (HBV) infection is one of the major causes of liver disease. Up to 400 million people have chronic HBVinfection worldwide. ${ }^{1,2}$ Patients with chronic hepatitis B (CHB) have a substantially increased risk of liver cirrhosis and hepatocellular carcinoma (HCC), which together result in approximately 1 million deaths worldwide every year. ${ }^{1,2}$ Antiviral treatment with nucleos(t)ide analogues (NAs) aims to interrupt the progression of CHB into advanced hepatic fibrosis and HCC. A high replication level of HBV DNA is a well-known risk factor for $\mathrm{HCC}^{3}$ and viral suppression with NAs reduces the risk of HCC development. ${ }^{4}$

Currently, several NAs are available for the treatment of CHB. They act on viral reverse transcriptase (RT) to inhibit HBV replication but do not kill the virus itself; thus, long-term continuous administration of NAs is necessary to maintain complete viral suppression. Because NAs require long-term use, there is a risk of emergence of drug-resistant mutants. In the case of lamivudine (LMV), genotypic resistance developed in up to $70 \%$ of CHB patients at year 5..$^{5-7}$ Although the currently available highpotency NAs such as entecavir (ETV) and tenofovir disoproxil fumarate (TDF) have a very low risk of genotypic resistance in NA-untreated patients, prior exposure to low-potency antivirals was reported to be associated with increased risk of developing ETV resistance. ${ }^{8}$ Furthermore, a number of CHB patients develop multidrug resistance mutations due to initial antiviral treatment with low-potency NAs followed by sequential rescue monotherapies. Thus, antiviral resistance mutations are still problematic even in the era of highly potent NAs.

\footnotetext{
Correspondence to: Kyun-Hwan Kim

Department of Pharmacology, Konkuk University School of Medicine, 120-1 Neungdong-ro, Gwangjin-gu, Seoul 05030, Korea

Tel: +82-2-2030-7833, Fax: +82-2-2049-6192, E-mail: khkim10@kku.ac.kr

Received on July 25, 2017. Revised on September 24, 2017. Accepted on September 24, 2017. Published online December 26, 2017

pISSN 1976-2283 eISSN 2005-1212 https://doi.org/10.5009/gnl17336

Doo Hyun Kim and Hong Seok Kang contributed equally to this work as first authors.

(c) This is an Open Access article distributed under the terms of the Creative Commons Attribution Non-Commercial License (http://creativecommons.org/licenses/by-nc/4.0) which permits unrestricted non-commercial use, distribution, and reproduction in any medium, provided the original work is properly cited.
} 
The rapid and accurate diagnosis of genotypic resistance is critical for immediate and appropriate rescue therapy. Various methods have been developed for the detection of HBV mutants, such as sequencing, dot blot, ${ }^{9}$ real-time polymerase chain reaction (PCR), ${ }^{10}$ the line probe assay, ${ }^{11}$ pyrosequencing, ${ }^{12}$ peptide nucleic acid array, ${ }^{13}$ restriction fragment length polymorphism, ${ }^{14}$ restriction fragment mass polymorphism (RFMP), ${ }^{15,16}$ and DNA microarray. ${ }^{14}$ However, most of these detection methods are lengthy and laborious; their pros and cons were previously reviewed.$^{17}$ In other words, there is no optimized technique available for detection of drug-resistant HBV mutants.

A drug-resistant HBV mutant results from a single nucleotide polymorphism (SNP) mutation in viral polymerase; a sequence with an SNP mutation is difficult to discriminate from the wildtype (WT) sequence when using pre-existing techniques. ${ }^{14}$ DNA microarray has been applied to detect SNP mutants of HBV by examining variations of the DNA sequence. ${ }^{14,18-20}$ Nevertheless, like other detection methods, conventional DNA microarray shows a high background signal, and identifying SNPs that induce drug resistance is a challenging task. The surface characteristics of microarray slides is critical point of DNA microarray performance. Conventional slides have too many functional groups and their spacing is irregular. To solve the spacing problem, dendron-modified slides (dendron slides) have been developed; they provide regular and wide interprobe spaces, referred to as mesospacing. ${ }^{21-23}$ The mesospacing of dendron slides could provide higher selectivity and sensitivity than those of other microarray slides, and discriminate the SNPs. ${ }^{24}$

In this study, we evaluated the accuracy and specificity of dendron slides for detection of genotypic resistance of HBV to NAs.

\section{MATERIALS AND METHODS}

\section{Preparation of HBV DNA}

We constructed 10 plasmids (replication-competent HBV1.2mer) encoding the WT HBV sequence or HBV sequences with mutations in the polymerase gene. These were produced by inserting HBV DNA into the PGEM-4z vector using XhoI and NcoI restriction enzyme sites. ${ }^{25,26} \mathrm{HBV}$ DNA was extracted using a virus spin kit (Qiagen GmbH, Hilden, Germany) from 200 $\mu \mathrm{L}$ of HBV-infected patient's serum. PCR was performed using not only viral DNA extracted from serum but also directly with serum samples without DNA extraction. PCR with sera was performed using 5x EzWay PCR buffer (KOMA Biotech Inc., Seoul, Korea).

\section{Patients}

For the validation about DNA microarray, 13 CHB patients who developed viral breakthrough during LMV, adefovir dipivoxil (ADV), or ETV therapy at the Konkuk University Medi- cal Center (Seoul, Korea) were included in this study. Serum of each patient was obtained after obtaining written informed consent. This study was approved by the Institutional Review Board of the Konkuk University Medical Center (IRB number: KUH1010277) and was conducted in accordance with the ethical guidelines.

The mean duration of LMV therapy until emergence of LMV resistance was 12 months. Serum from patient \#6 was collected in February 2006, May 2007, and March 2008 (designated as P6-1, P6-2, and P6-3, respectively) analyzed by DNA microarray using dendron slides, RFMP, and sequencing. Patient \#6 was treated with LMV as an initial treatment for HBV. However, viral breakthrough developed after 15 months of LMV therapy. ADV was added to LMV treatment and the viral DNA level steadily declined with this combination therapy. Serum from patient \#7 was obtained in October 2006, November 2007, and February 2009 (designated as P7-1, P7-2, and P7-3, respectively) and analyzed as above. Patient \#7 was treated with LMV as an initial treatment for HBV but experienced a viral breakthrough after 13 months of LMV therapy. After switching to ADV, HBV DNA levels decreased. However, another viral breakthrough appeared after 8 months of ADV treatment. ETV was added to ADV, and the viral DNA level steadily decreased. In total, we analyzed 11 serum samples from seven patients. Six serum samples were received from the tissue bank of the Konkuk University Hospital (Seoul, Korea). RFMP analysis was performed at the Konkuk University Hospital and sequence analysis was performed at Macrogen (Macrogen Inc., Seoul, South Korea).

\section{DNA microarray slides and capture probes}

9-Amine dendron slides was obtained from NSB Postech (NSB Postech Inc., Pohang, Korea). A $9 \mathrm{~mm} \times 9 \mathrm{~mm}$ square-shaped frame seal was purchased from Bio-Rad (Bio-Rad Laboratories, Inc., Hercules, CA, USA). Aldehyde, aldehyde2, and epoxy slides were purchased from SCHOTT (SCHOTT AG., Mainz, Germany), TeleChem International (TeleChem International Inc., Portland, OR, USA), and Corning (Corning Inc., Corning, NY, USA), respectively. Capture probes used in this study are listed in Table 1. All capture probes were synthesized by Bioneer Company (Bioneer Corp., Daejeon, Korea) or Thermo (Thermo Fisher Scientific Corp., Waltham, MA, USA).

\section{Crosslinking of capture probes to slides}

Di-(N-succinimidyl) carbonate was used as a linker molecule between the dendron molecules and the capture probes. Capture probes $(20 \mathrm{pM})$ were spotted on dendron glass slides as described previously. ${ }^{23}$ Capture probes in spotting buffer solution were printed on slides using a microarrayer (QArray; Genetix Ltd., New Milton, UK). After removing unbound capture probes, slides were dried with nitrogen steam and stored at $4^{\circ} \mathrm{C}$. 


\section{Amplification of target DNA}

PCR primers for amplifying HBV DNA were designed to include the sequences encoding the amino acids that determine drug resistance and generated a 440-base pair product (Table 1). The 5' ends of all primers were labeled with cy3. The reaction mixture $(30 \mu \mathrm{L})$ contained $3 \mu \mathrm{L}$ of $10 \times$ buffer, $1 \mu \mathrm{L}$ of each of the four dNTPs (10 mM each), $0.6 \mu \mathrm{L}$ of Taq polymerase (5 units $/ \mu \mathrm{L}$ ), $1 \mu \mathrm{L}$ of cy3-forward primer (BVF, $5 \mathrm{pmol} / \mu \mathrm{L}), 1 \mu \mathrm{L}$ of reverse primer (BVR, $50 \mathrm{pmol} / \mu \mathrm{L}$ ), $1 \mu \mathrm{L}$ of sample (plasmid or extracted HBV DNA), and $22.4 \mu \mathrm{L}$ of distilled water. For direct PCR amplification using serum without virus purification, the PCR mixture contained $6 \mu \mathrm{L}$ of 5x PCR buffer (KOMA Biotech Inc.), $1 \mu \mathrm{L}$ of each of the four dNTPs (10 mM each), $0.6 \mu \mathrm{L}$ of Taq polymerase ( 5 units $/ \mu \mathrm{L}), 1 \mu \mathrm{L}$ of BVF $(5 \mathrm{pmol} / \mu \mathrm{L}), 1 \mu \mathrm{L}$ of BVR ( $50 \mathrm{pmol} / \mu \mathrm{L}$ ), $1 \mu \mathrm{L}$ of serum, and $19.4 \mu \mathrm{L}$ of distilled water. PCR was carried out as follows: heating at $94^{\circ} \mathrm{C}$ for 5 minutes; 40 cycles of 1 minute at $94^{\circ} \mathrm{C}, 1$ minute at $52^{\circ} \mathrm{C}, 1$ minute at

Table 1. Sequences of Hepatitis B Virus Primers and Probes Used in This Study

\begin{tabular}{lll}
\hline & \multicolumn{1}{c}{ Sequence $\left(5^{\prime} \rightarrow 3^{\prime}\right)$} & Length \\
\hline Primer & & \\
BVF & Cy3-TTT CCC TCT TGT TGC TGT ACA AAA CC & 26 \\
BVR & Cy3-TGA CAT ACT TTC CAA TCA ATA GGT CTA & 27 \\
Probe & & \\
rtL180 & Amine-TCA GTC CGT TTC TCC TNG CTC & 21 \\
rtL180M & Amine-CTC AGT CCG TTT CTC ATG G & 19 \\
rtA181 & Amine-GTC CGT TTC TCC TGG CTC A & 19 \\
rtA181T & Amine-CAG TCC GTT TCT CCT GAC TCA & 19 \\
rtA181V & Amine-AGT CCG TTT CTC CTG GTT CA & 20 \\
rtM204 & Amine-GTT TGG CTT TCA GTT ATA TGG AT & 23 \\
rtM204I & Amine-TGT TTG GCT TTC AGT TAT ATT GAT G & 25 \\
rtM204V & Amine-GTT TGG CTT TCA GTT ATG TGG & 21 \\
rtN236 & Amine-GTC TTT GGG TAT ACA TTT GAA CCC & 24 \\
rtN236T & Amine-CTT TGG GTR TAC AKT TR ACC CCT & 23 \\
rtM250 & Amine-GCT ACT CCC TTA ACT TCA TGG G & 22 \\
rtM250V & Amine-GGC TAC TCC CTT AAC TTC GTG G & 22 \\
rt169 & Amine-CCC GGG CTT TCG CAA GAT TCC & 21 \\
rt169T & Amine-GGG CTT TCG CAA GAC TCC & 18 \\
rtV173 & Amine-GCA AGA TTC CTA TGG GAG TGG & 21 \\
rtV173L & Amine-GCA AGA TTC CTA TGG GAT TGG & 21 \\
rtT184 & Amine-TTT CTC CTG GCT CAG TTT ACT AG & 23 \\
rtT184A & Amine-CTC CTG GCT CAG TTT GCT AG & 20 \\
rtT184L & Amine-TTT CTC ATG GCT CAG TTT CTT AG & 23 \\
rtA194 & Amine-TTC AGT GAT TCG YAG GGC TT & 20 \\
rtS202 & Amine-CCC ACT GTT TGG CTT TCA GTT AT & 23 \\
rtS202G & Amine-ACT GTT TGG CTT TCA TTT A & 19 \\
\hline
\end{tabular}

$72^{\circ} \mathrm{C}$, and finally 7 minutes at $72^{\circ} \mathrm{C}$.

\section{Hybridization and scanning}

After PCR, the mixture was heated at $94^{\circ} \mathrm{C}$ for 5 minutes and chilled on ice. PCR mixtures were transferred to a glass slide which with immobilized capture probes and incubated on a thermoblock at $50^{\circ} \mathrm{C}$ for 2 hours. After hybridization, slides were washed with distilled water, dried by nitrogen gas, and scanned with a laser scanner (ScanArray PLUS; PerkinElmer. Inc., Waltham, MA, USA). Quantitative data analysis was performed using QantArray software (PerkinElmer, Inc.).

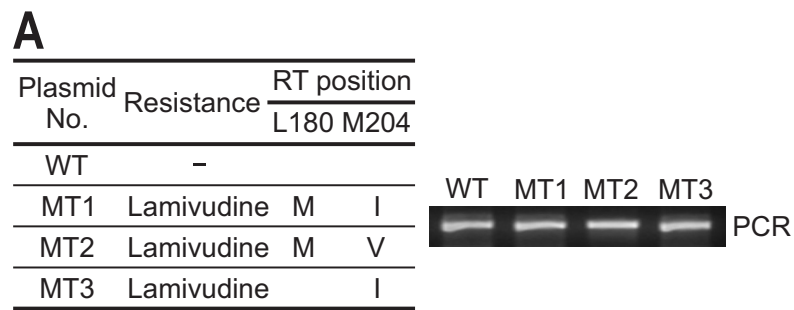

\section{B}

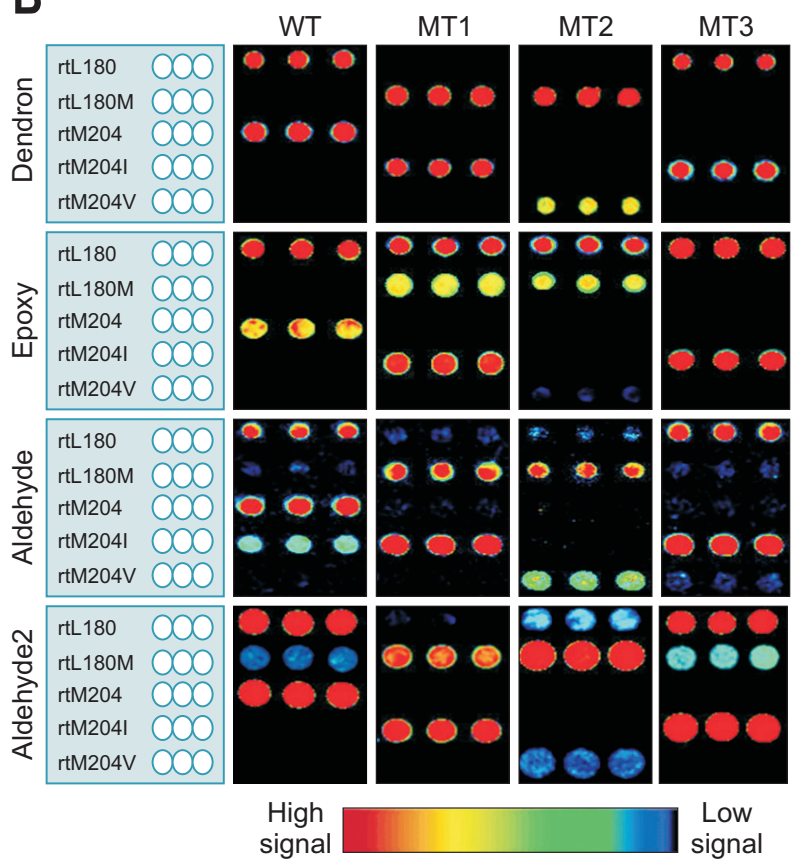

Fig. 1. Comparison of the specificity of dendron and conventional slides in the detection of hepatitis B virus drug-resistance mutations. Five types of probes were printed on each slide. (A) Validation of the efficiency of the polymerase chain reaction (PCR) amplification of the probe-binding regions. The constructed plasmids encoding wild-type (WT) polymerase or polymerase with mutations at positions rt180 and rt204 are indicated. (B) Comparison of the specificity of dendron and conventional slides coated with epoxy or two types of aldehydes. The layout of the probe positions is indicated on the left. Each slide had five probes in triplicate. The indicated PCR products were hybridized to each slide. After washing, the fluorescence signal intensity was measured. Color gradation indicates the signal intensity.

RT, reverse transcriptase. 
A

Plasmid copy number
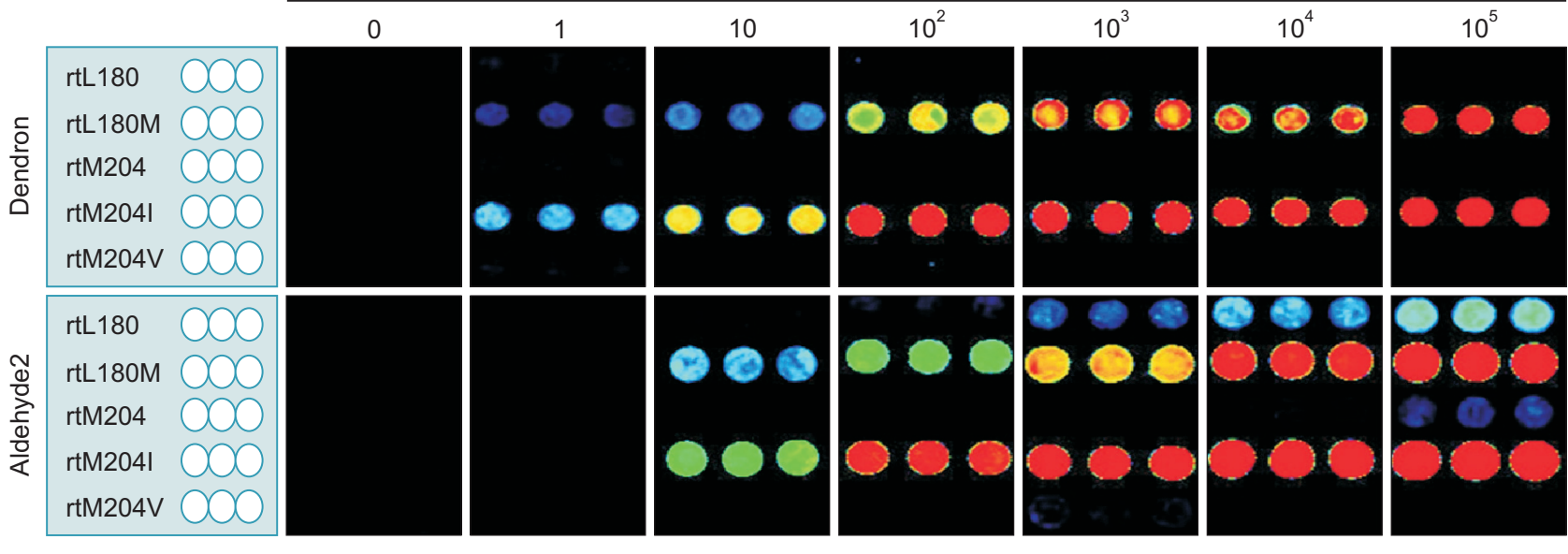

B
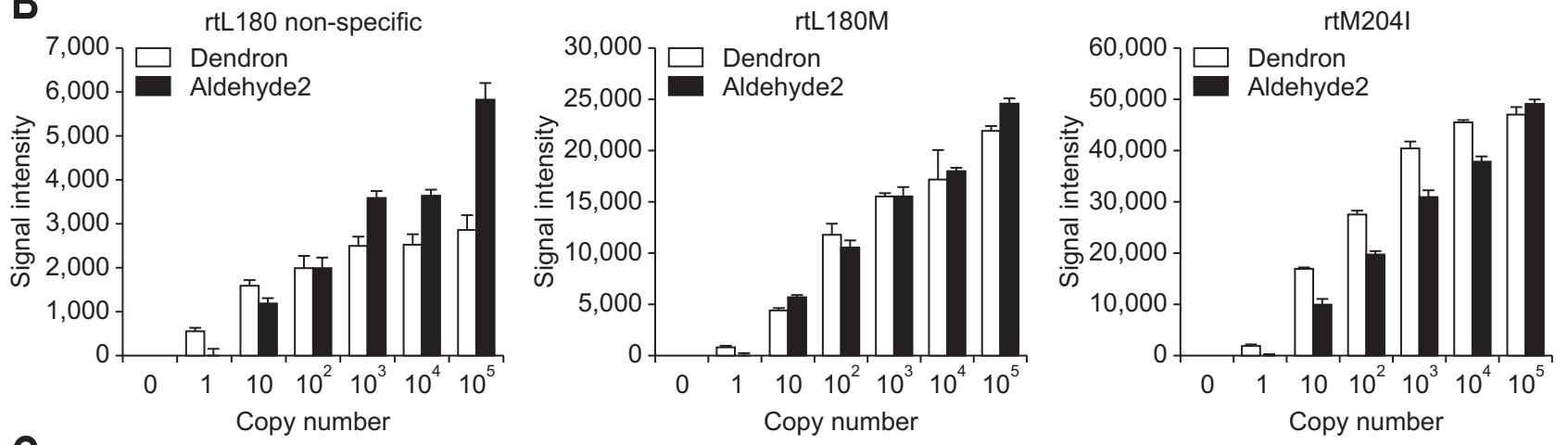

C

Wild-type: mutant ratio

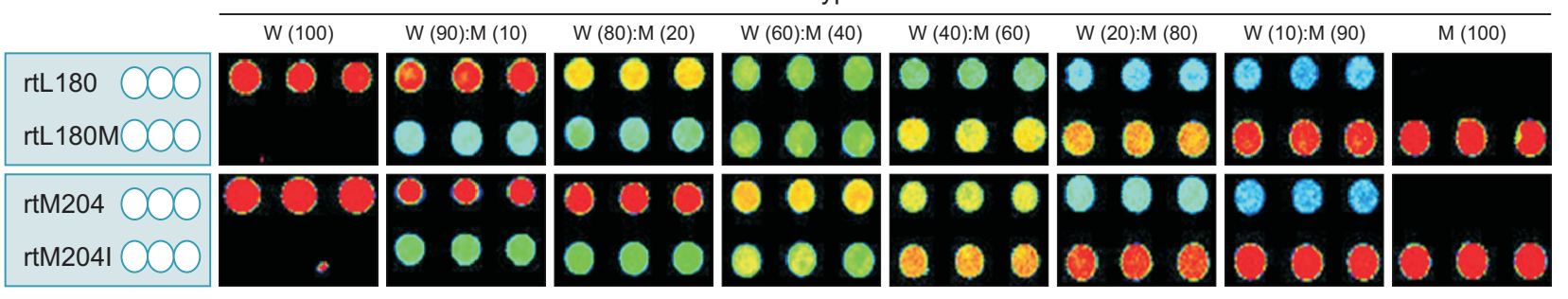

D
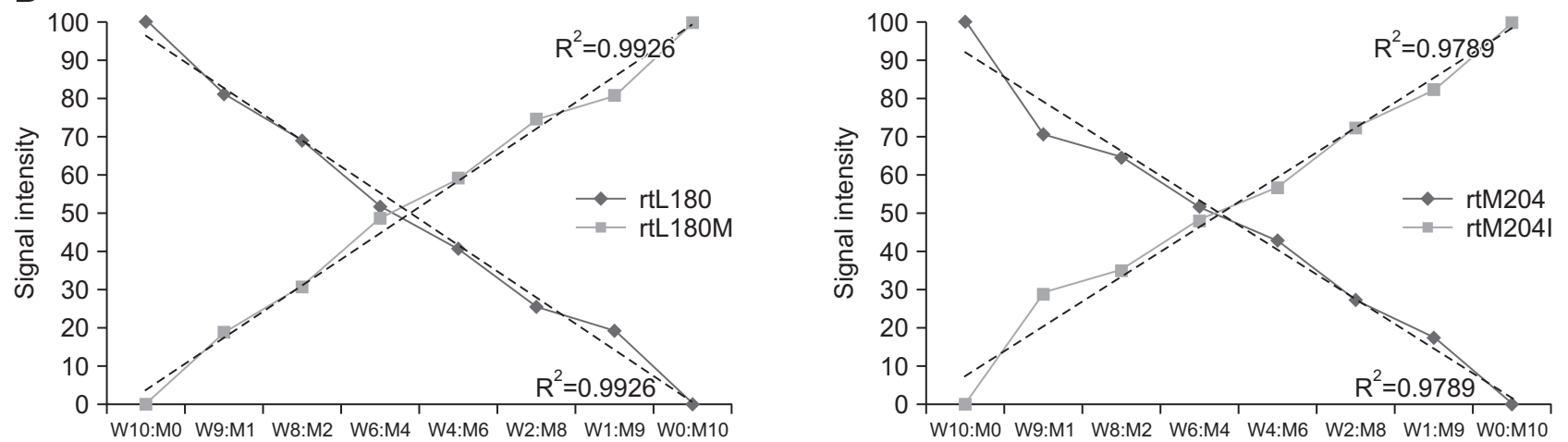

Fig. 2. Sensitivity and selectivity of a dendron slide in the detection of drug-resistant hepatitis B virus mutants. (A) Sensitivity test of a dendron slide compared to that of an aldehyde2 slide. The indicated five plasmids containing wild-type (WT) polymerase or polymerase harboring mutations at positions rt180 and rt204 were serially diluted. Each sample containing the indicated plasmid copy number was amplified by polymerase chain reaction (PCR) and hybridized. (B) Scanned signal intensities obtained using the quantification mode of the software to operate the ScanArray scanner are plotted for comparison. (C, D) Selectivity test of a dendron slide. Mutant plasmids were mixed with the WT plasmid at the indicated ratios; $10^{5}$ plasmid copies were used for each PCR amplification. W, WT plasmid; M, mutant plasmid; percentage of the WT or mutant plasmid. 
A

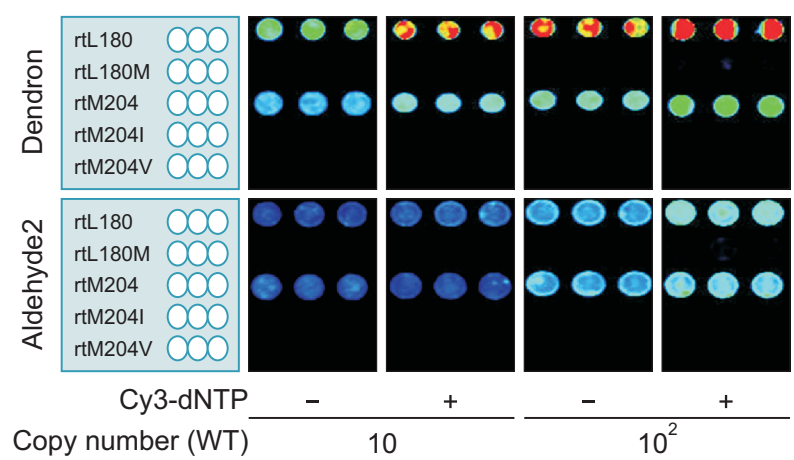

B

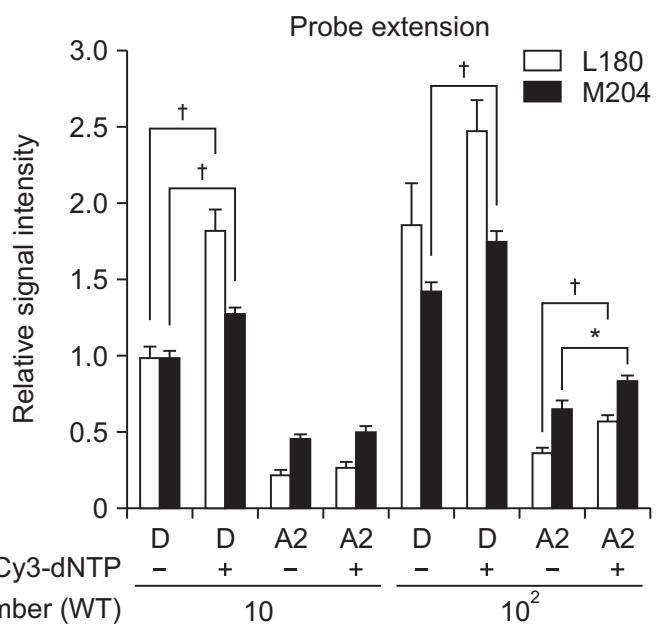

Fig. 3. Signal enhancement in a dendron slide by the addition of cy3-labeled dNTPs during hybridization. (A) Indicated hepatitis B virus (HBV) plasmids (10 or $10^{2}$ copies) were used for the polymerase chain reaction amplification. The cy3-dNTPs (a mixture of cyanine-3-labeled dATP, dTTP, dGTP, and dCTP) were added at a final concentration of $1 \mathrm{nmol} / \mu \mathrm{L}$. (B) Relative signal intensities compared to the intensity obtained using 10 copies of the HBV plasmid on a dendron slide.

D, dendron slide; A2, aldehyde2 slide. ${ }^{*} \mathrm{p}<0.02 ;{ }^{\dagger} \mathrm{p}<0.001$.

A

Patient number

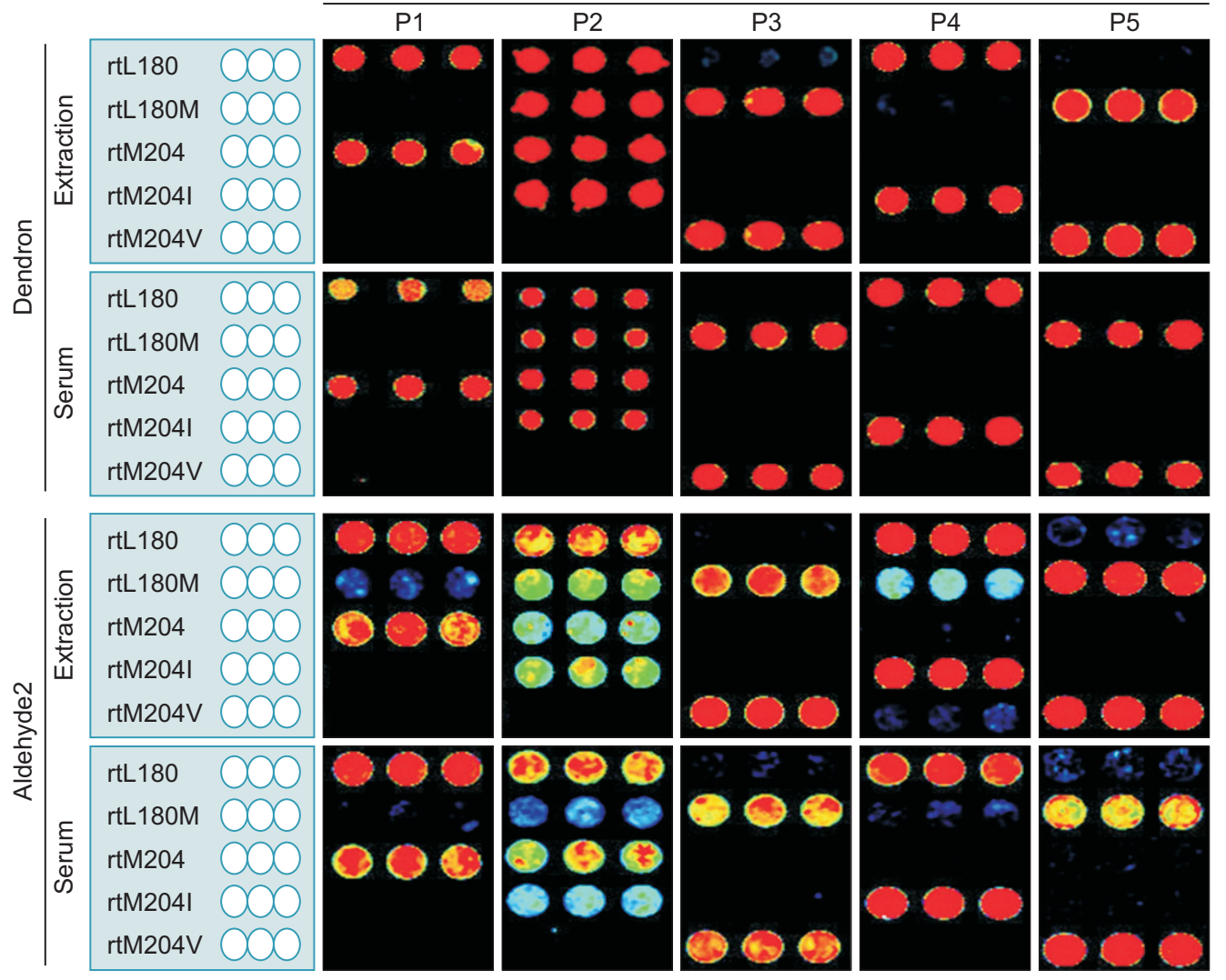

Fig. 4. Direct detection of drug-resistance mutations in patient sera without virus purification, and validation of a dendron slide using sequencing and restriction fragment mass polymorphism (RFMP). (A) Comparison of microarray detection of polymerase chain reaction (PCR) products amplified from purified hepatitis B virus (HBV) DNA and directly from serum. Sera from five chronic HBV (CHB) patients (P1-P5) were used. PCR amplification was performed using 5'-end-labeled cy-3 primers. Each PCR product was analyzed using dendron or aldehyde2 slides. During hybridization, cy-3 dNTPs were added to increase the signal intensity. (B) The clinical courses of two CHB patients and the timing of the serum sampling. The serum samples obtained at each time point were analyzed using a dendron chip, RFMP, and sequencing. Sequencing electropherograms of the rt180 and rt204 positions are presented (L, CTG; M, ATG; I, ATT; V, GTG). LMV, lamivudine; ADV, adefovir; ETV, entecavir. 


\section{RESULTS}

\section{Comparison of dendron slides with conventional slides} for detection of drug-resistant HBV mutants

To compare the specificity of dendron slides with that of conventional slides, we first generated four HBV plasmids encoding WT or mutated polymerase, and confirmed that the PCR efficiency of those clones was similar (Fig. 1A).

The dendron slides were designed as shown in Fig. 1B, and the five capture probes were immobilized in triplicate on each slide. As shown in Fig. 1B, only a dendron slide showed the perfectly matched signals against WT and the three mutants without any non-specific binding. In contrast, an epoxy slide could not discriminate between the rtL180L and rtL180M mutants and could not detect rtM204V. Similarly, aldehyde and aldehyde2 slides could not distinguish rtM204M from rtM204I and rtL180M from rtL180L, respectively. Non-specific signals were more frequent on aldehyde slides. These results indicated that dendron slides are superior to conventional slides in both specificity and sensitivity for detection of drug-resistant HBV mutations.

\section{Sensitivity and selectivity of dendron slides for detection of drug-resistant HBV mutants}

Since an aldehyde2 slide and a dendron slide showed similar signal intensity, we compared the sensitivity and selectivity of these two slides. First, we determined their sensitivity using a serial dilution of a plasmid encoding the rtL180M/rtM204I double mutant. The lower limit of detection of the dendron slide was 1 copy/ $\mu \mathrm{L}$ of HBV DNA, whereas it was 10 copies/ $\mu \mathrm{L}$ for the aldehyde2 slide (Fig. 2A). Non-specific signals, especially at position rt180, were increased proportionally to the copy number on the aldehyde2 slide (Fig. 2A and B). This result indicates that the dendron slide was 10 times more sensitive than the
B
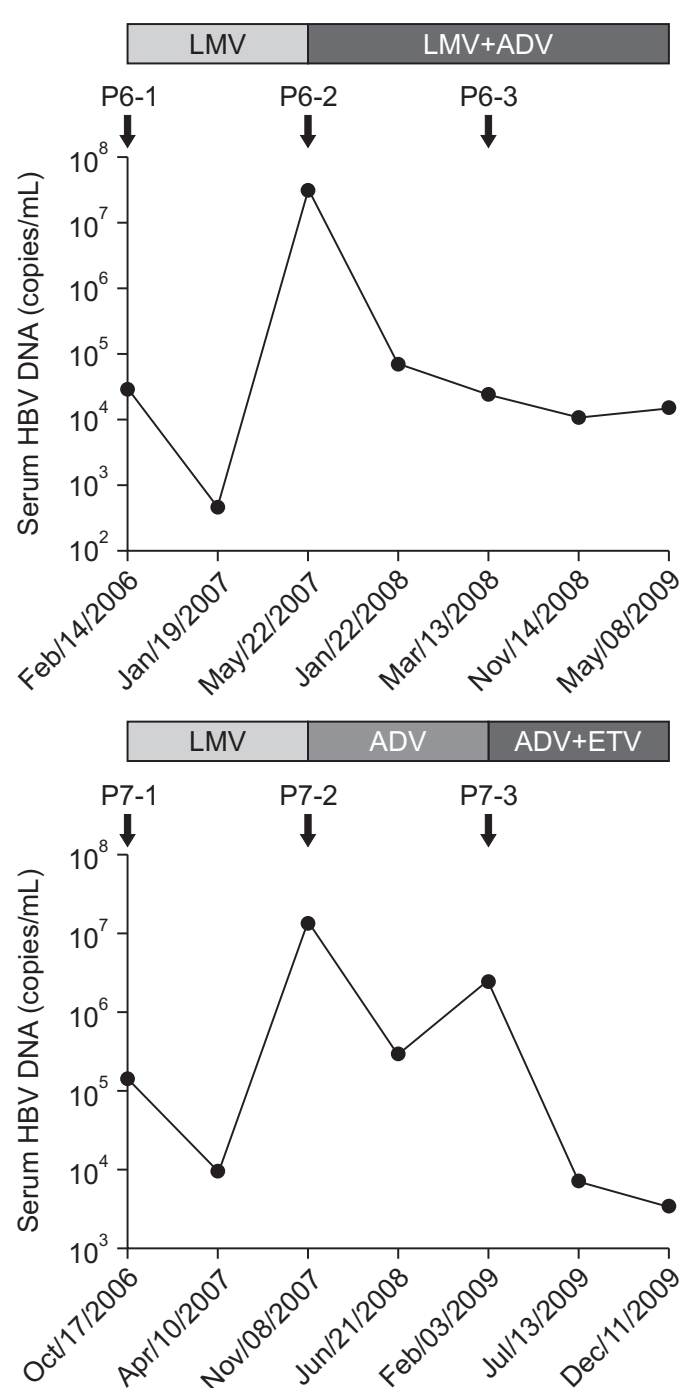

\begin{tabular}{cc}
\multicolumn{2}{c}{ RFMP } \\
\hline $180 \quad 204$ \\
\hline
\end{tabular}
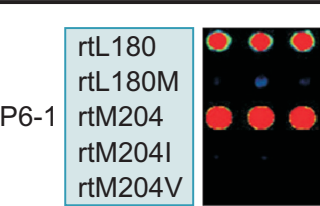

L180

\section{M204}

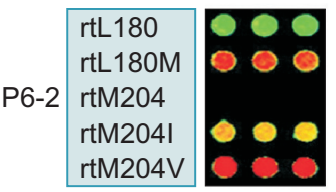

L180M

M204I\&V

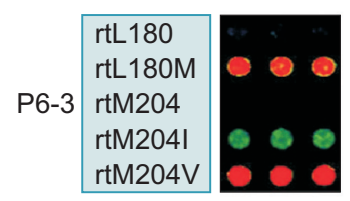

L180M

M204V

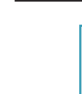

P7-1

$\mathrm{rtL} 180$

rtL180M

rtM204I

rtM204V

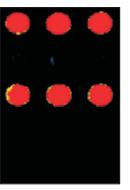

L180

M204

P7-2

rtL180

rtL180M

rtM204

rtM204I

rtM204V

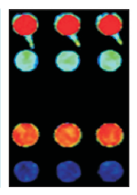

L180

rtL180

rtL180M

P7-3

rtM204

rtM204I

rtM204V

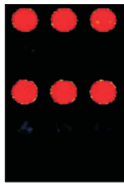

L180
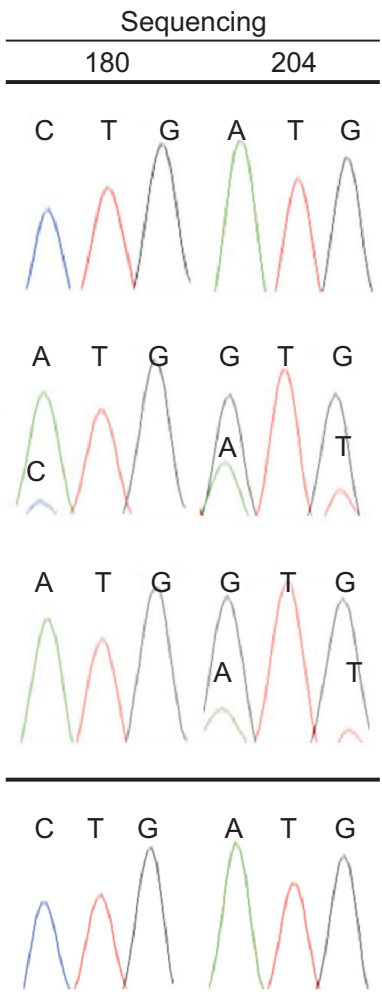

M204I

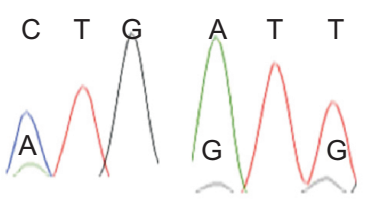

M204

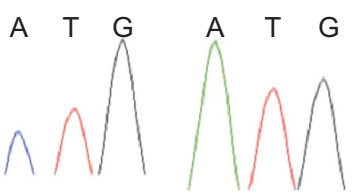

Fig. 4. Continued. 
aldehyde2 slide, and had good selectivity.

Next, we determined the selectivity of the dendron slide using a template DNA mixed with either rtL180 and rtL180M, or rtM204 and rtM204I at known ratios (Fig. 2C and D). The quantitated signals of both mixtures showed linear changes between samples and were well correlated with the expected data. These results indicate that dendron slides can be applied for semiquantitative detection of drug-resistant HBV mutations.

\section{Enhanced sensitivity of detection of drug-resistant HBV by addition of cy3-labled dNTPs during hybridization}

Since a previous study demonstrated that signal amplification for detection of human papilloma virus is increased by branched DNA formation during hybridization, ${ }^{27}$ we tested whether the detection signal in case of HBV mutations can be also similarly enhanced.

The signal intensity of rtL180 on a dendron slides was five times that of an aldehyde2 slide without cy3-dNTPs (Fig. 3A). However, addition of cy3-dNTPs during hybridization resulted in a significant increase in signal intensity on the dendron slide (2-fold increase in rtL180 detection at 10 copies). However, there was no significant increase in signal intensity on the aldehyde2 slide when cy3-dNTPs were added (Fig. 3B). These differences may be due to the nature of regular and wide interprobe spaces of dendron slides. ${ }^{21-23}$ These results indicate that the addition of cy3-dNTPs during hybridization can significantly increase the intensity of signals on dendron slides.

\section{Direct detection of drug-resistant mutants from patients' sera without purification of viral DNA and validation of dendron slides using other conventional methods}

To evaluate whether virus purification is necessary for detection with dendron slides, we compared purified HBV DNA and patient serum as PCR templates. We found that, when a dendron slide was used, the results were similar with patient's serum and with purified HBV DNA. However, an aldehyde2 slide showed considerable non-specific binding and lower detection signals both with purified HBV DNA and patient serum (Fig. 4A).

To validate the results with patient's serum obtained by using a dendron slide, we compared them with those of RFMP and sequencing. Six serum samples from two CHB patients (P61, P6-2, P6-3, P7-1, P7-2, and P7-3) were subjected to analysis using a dendron slide, RFMP, and direct sequencing (Fig. $4 \mathrm{~B})$. The result of the dendron slide revealed the emergence of rtL180M and rtM204I/V mutations in the P6-2 sample, which corresponds to the clinical viral breakthrough with LMV. This result was concordant with sequencing data but not with that of RFMP. The emergence of rtL180M and rtM204I/V mutations in the P7-2 sample was also identified; it also corresponds to the clinical viral breakthrough with LMV. When the level of serum HBV DNA was decreased by treatment with ADV+ETV, the LMV-resistance mutations were no longer detectable. This result was concordant with that of RFMP and sequencing data.

HBV mutations identified from dendron slides using $11 \mathrm{CHB}$ serum samples were mostly concordant with those of RFMP and sequencing (Table 2). The results for P6-2, P6-3, and P7-2 were slightly discordant with RFMP data but matched sequencing

Table 2. Hepatitis B Virus Mutations Identified in Patient Sera Using a Dendron Slide, RFMP, and Sequencing

\begin{tabular}{|c|c|c|c|c|}
\hline \multirow{2}{*}{$\begin{array}{c}\text { Sample } \\
\text { no. }\end{array}$} & \multirow{2}{*}{$\begin{array}{l}\text { Viral titer, } \\
\text { copy } / \mathrm{mL}\end{array}$} & \multirow{2}{*}{ Analysis method } & \multicolumn{2}{|c|}{ RT position } \\
\hline & & & rtL180 & rtM204 \\
\hline \multirow[t]{3}{*}{$\mathrm{P} 1$} & $1.80 \times 10^{7}$ & Dendron chip & - & - \\
\hline & & RFMP & - & - \\
\hline & & Sequencing & - & - \\
\hline \multirow[t]{3}{*}{$\mathrm{P} 2$} & $3.88 \times 10^{7}$ & Dendron chip & $\mathrm{L}, \mathrm{M}$ & $\mathrm{M}, \mathrm{I}$ \\
\hline & & RFMP & $\mathrm{L}, \mathrm{M}$ & $\mathrm{M}, \mathrm{I}$ \\
\hline & & Sequencing & $\mathrm{L}, \mathrm{M}$ & $\mathrm{M}, \mathrm{I}$ \\
\hline \multirow[t]{3}{*}{ P3 } & $2.43 \times 10^{7}$ & Dendron chip & M & V \\
\hline & & RFMP & M & V \\
\hline & & Sequencing & M & V \\
\hline \multirow[t]{3}{*}{$\mathrm{P} 4$} & $4.50 \times 10^{6}$ & Dendron chip & - & I \\
\hline & & RFMP & - & I \\
\hline & & Sequencing & - & I \\
\hline \multirow[t]{3}{*}{ P5 } & $8.21 \times 10^{6}$ & Dendron chip & M & V \\
\hline & & RFMP & M & V \\
\hline & & Sequencing & M & V \\
\hline \multirow[t]{3}{*}{ P6-1 } & $3.04 \times 10^{4}$ & Dendron chip & - & - \\
\hline & & RFMP & - & - \\
\hline & & Sequencing & - & - \\
\hline \multirow[t]{3}{*}{ P6-2 } & $3.13 \times 10^{7}$ & Dendron chip & $\mathrm{L}, \mathrm{M}$ & $\mathrm{V}, \mathrm{I}$ \\
\hline & & RFMP & M & V, I \\
\hline & & Sequencing & $\mathrm{L}, \mathrm{M}$ & V, I \\
\hline \multirow[t]{3}{*}{ P6-3 } & $6.77 \times 10^{4}$ & Dendron chip & M & $\mathrm{V}, \mathrm{I}$ \\
\hline & & RFMP & M & V \\
\hline & & Sequencing & M & V \\
\hline \multirow[t]{3}{*}{ P7-1 } & $1.43 \times 10^{5}$ & Dendron chip & - & - \\
\hline & & RFMP & - & - \\
\hline & & Sequencing & - & - \\
\hline \multirow[t]{3}{*}{ P7-2 } & $1.35 \times 10^{7}$ & Dendron chip & $\mathrm{L}, \mathrm{M}$ & $\mathrm{V}, \mathrm{I}$ \\
\hline & & RFMP & - & I \\
\hline & & Sequencing & $\mathrm{L}, \mathrm{M}$ & $\mathrm{V}, \mathrm{I}$ \\
\hline \multirow[t]{3}{*}{ P7-3 } & $2.53 \times 10^{6}$ & Dendron chip & - & - \\
\hline & & RFMP & - & - \\
\hline & & Sequencing & - & - \\
\hline
\end{tabular}

RFMP, restriction fragment mass polymorphism; RT, reverse transcriptase; L, CTG; M, ATG; I, ATT; V, GTG. 
data. Considering that sequencing results are most reliable, our results suggest that dendron slides can be used as a diagnostic technique for the detection of drug-resistant HBV.

\section{Simultaneous and direct detection of LMV-, ADV-, and ETV-resistant mutants from patients' sera on a single dendron-modified array chip}

Since we confirmed that dendron slides are capable of detecting the LMV-resistance mutant HBV in sera from $\mathrm{CHB}$ patients (Fig. 4), we further investigated whether it is possible to simultaneously discriminate between LMV-, ADV-, and ETV-resistance mutations on the same slide. We obtained a total of 22 probes (nine for WT; four for LMV-resistant mutants; three for ADVresistant mutants; and six for ETV-resistant mutants) (Table 1) through trial and error and finally designed the array chip shown in Fig. 5A. To validate this chip and probe, we construct- ed six more $\mathrm{HBV}$ mutants that harbored various combinations of drug-resistance mutations (Fig. 5B). As shown in Fig. 5C, a dendron chip perfectly distinguished each mutation without any non-specific binding. The WT area on the left side of the chip shows which positions of HBV RT are mutated, and each drugresistance area in the middle of the chip shows what the specific drug-resistance mutations are.

We further validated the dendron chip using the sera of CHB patients (Fig. 6A). Chip analysis revealed that serum sample B1247 contains a complete WT HBV (all positive detection in the left box and no signals in the middle and right boxes). HBV detected in sera B5656, B5454, and B8373 showed an ETV-resistant pattern (positive in the right box). All sera except B1247 harbored the LMV-resistance mutations (rtL180M and rtM204I/ V). To confirm these results, we compared the chip data with HBV sequences analyzed using RFMP and sequencing (Fig. 6B).
A

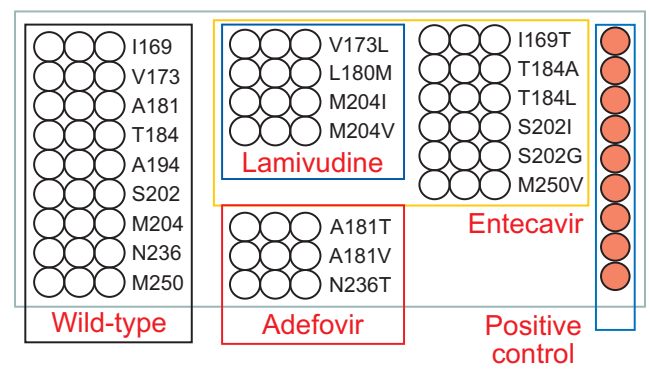

C

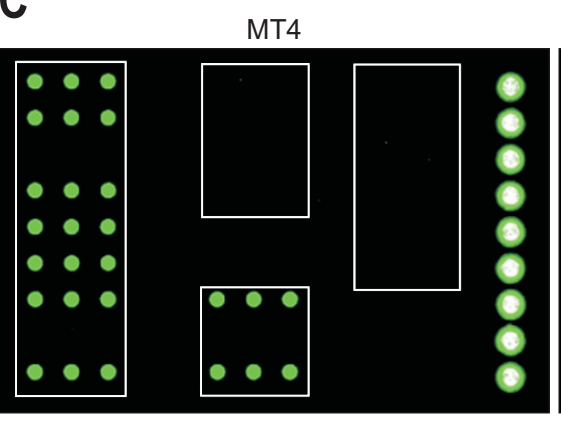

MT7

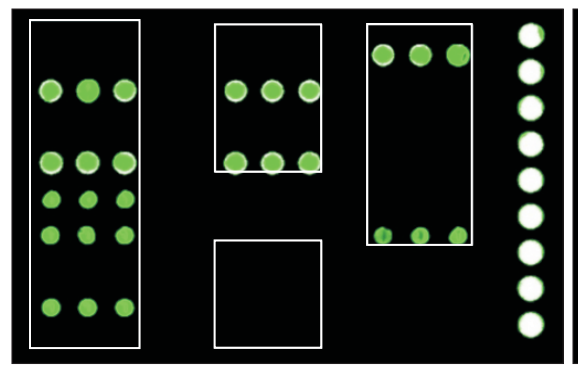

B

\begin{tabular}{lllllllllll}
\hline \multirow{2}{*}{$\begin{array}{c}\text { Plasmid } \\
\text { No. }\end{array}$} & Resistance & \multicolumn{8}{c}{ RT position } \\
\cline { 2 - 12 } MT4 & Adefovir & - & - & - & T & - & - & - & T & - \\
\hline MT5 & Lamivudine & - & L & - & - & - & - & I & - & - \\
\hline MT6 & Entecavir & T & M & M & - & A & - & V & - & - \\
\hline MT7 & Entecavir & T & - & M & - & - & - & V & - & V \\
\hline MT8 & Entecavir & - & - & M & - & L & - & V & - & V \\
\hline MT9 & Entecavir & - & - & M & - & - & G & V & - & - \\
\hline
\end{tabular}

MT5

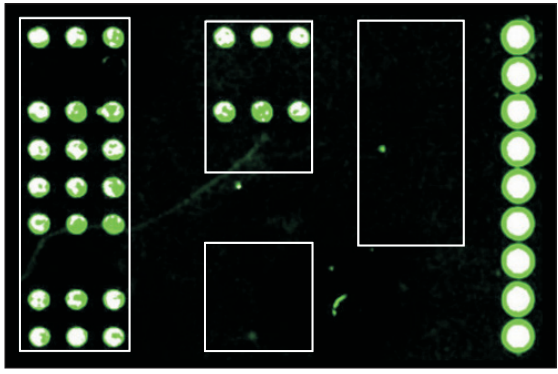

MT8

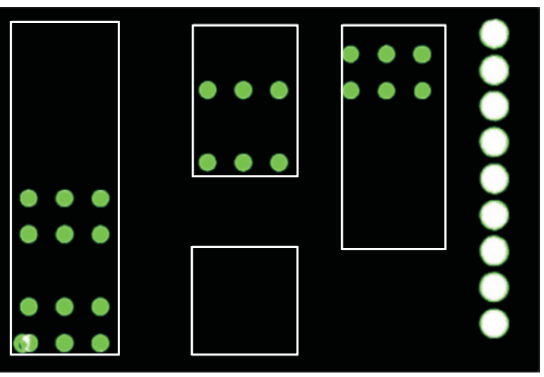

MT9
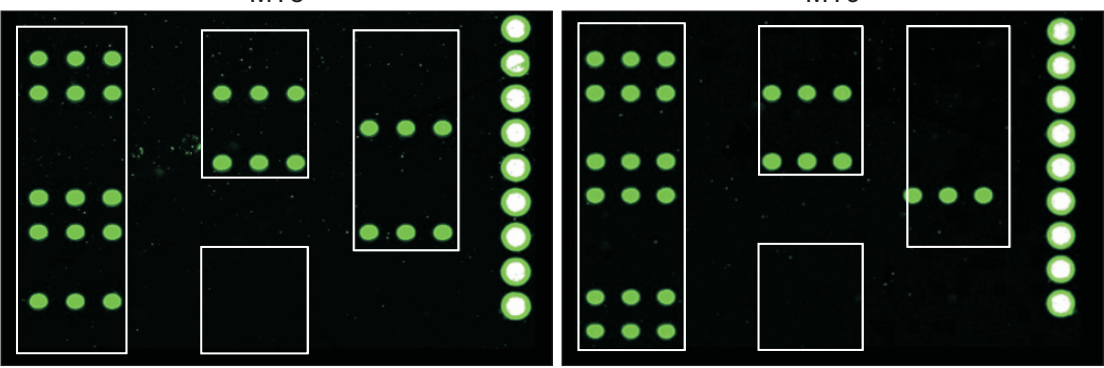

Fig. 5. Design and validation of a dendron-modified microarray capable of simultaneously detecting several drug-resistance mutations on a single slide. (A) Microarray design. Each drug-resistance mutation is presented in a box. (B) Construction of drug-resistant mutants. A combination of known resistance mutations for each drug was used for cloning. (C) Specificity of the dendron chip. Each amplified polymerase chain reaction product was hybridized to a dendron chip. After washing, the fluorescence signal intensity was measured. During hybridization, cy-3 dNTPs were added to increase the signal intensity.

RT, reverse transcriptase. 
A

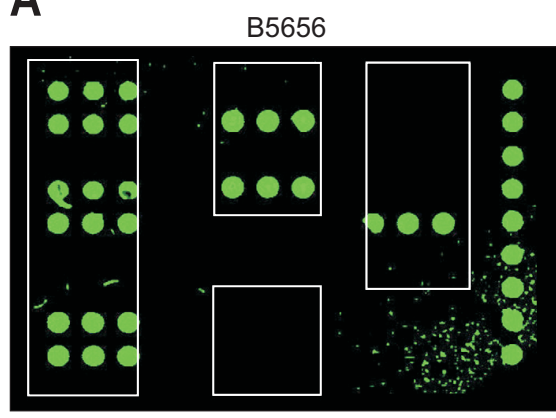

B1752

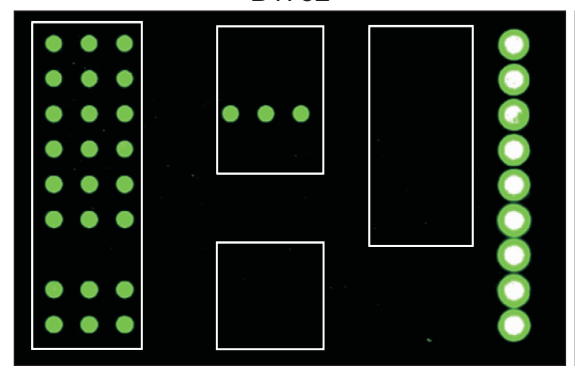

B5454

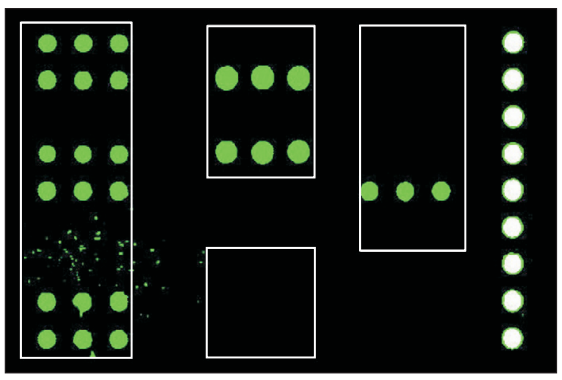

B2983

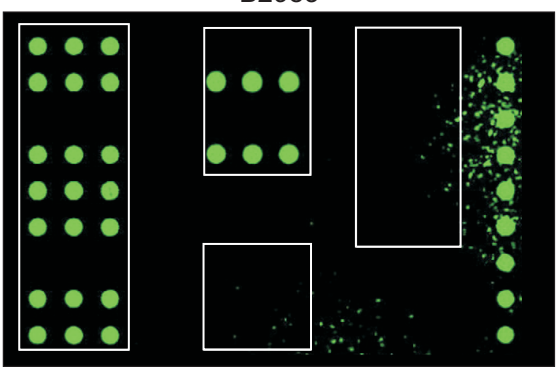

B8373

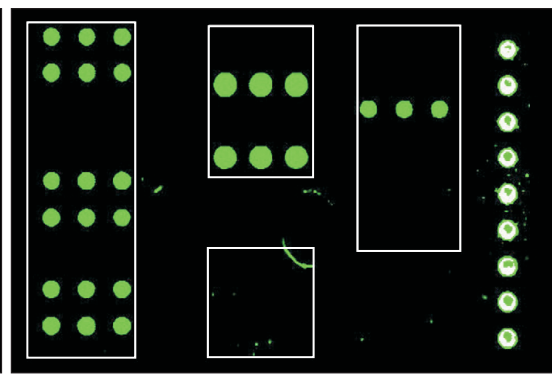

B1247

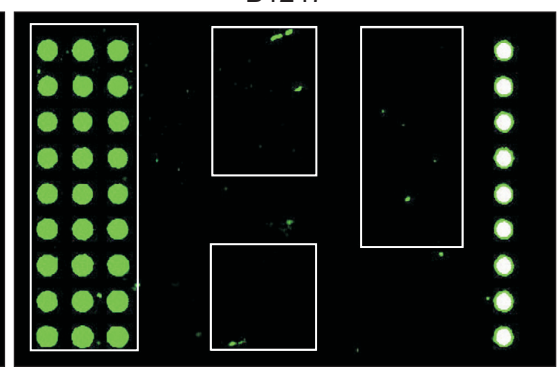

\section{B}

\begin{tabular}{|c|c|c|c|c|c|}
\hline \multirow{2}{*}{$\begin{array}{c}\text { Serum } \\
\text { No. }\end{array}$} & \multirow{2}{*}{ Method } & \multicolumn{4}{|c|}{ RT position } \\
\hline & & L180 & T184 & S202 & M204 \\
\hline \multirow{3}{*}{ B5656 } & Dendron chip & $\mathrm{M}$ & - & $G$ & $\mathrm{~V}$ \\
\hline & RFMP & ND & - & $\mathrm{G}$ & - \\
\hline & Sequencing & $\mathrm{M}$ & - & $G: S=9: 1$ & V \\
\hline \multirow{3}{*}{ B5454 } & Dendron chip & $\mathrm{M}$ & - & $G$ & V \\
\hline & RFMP & ND & $\mathrm{T}: \mathrm{A}=5: 1$ & $G: S=5: 1$ & - \\
\hline & Sequencing & $\mathrm{M}$ & $\mathrm{T}: \mathrm{A}=9: 1$ & $\mathrm{G}: S=9: 1$ & V \\
\hline \multirow{3}{*}{ B8373 } & Dendron chip & $M$ & $\mathrm{~L}$ & - & $\mathrm{V}$ \\
\hline & RFMP & ND & L & - & V \\
\hline & Sequencing & $\mathrm{M}$ & $\bar{L}$ & - & V \\
\hline \multirow{3}{*}{ B1752 } & Dendron chip & - & - & - & I \\
\hline & RFMP & - & - & - & I \\
\hline & Sequencing & - & - & - & I \\
\hline \multirow{3}{*}{ B2983 } & Dendron chip & $M$ & - & - & $\mathrm{V}$ \\
\hline & RFMP & $M$ & - & - & V \\
\hline & Sequencing & M & - & - & V \\
\hline \multirow{3}{*}{ B1247 } & Dendron chip & - & - & - & - \\
\hline & RFMP & - & - & - & - \\
\hline & Sequencing & - & - & - & - \\
\hline
\end{tabular}

We found a perfect match between sequencing and chip results, whereas the detection of rtM204V by RFMP was discordant with both the chip and sequencing data in two patients. These results suggest that dendron-modified arrays can be used for diagnostic purposes for the detection of all known drug-resistant HBV mutants.

\section{DISCUSSION}

In this study, we developed a simple and accurate method for simultaneous detection of genotypic resistance of HBV to LMV, ADV, and ETV using a dendron-modified microarray. We found that dendron slides are superior to conventional microarray slides in detecting the SNPs of drug-resistant HBV mutants.
Fig. 6. Direct detection of several nucleos(t)ide analogue-resistance mutations in patient sera on a single dendron chip and validation using sequencing and restriction fragment mass polymorphism (RFMP) data. (A) Sera from six chronic HBV patients were directly used for polymerase chain reaction (PCR), and the PCR products were analyzed using a dendron slide. During hybridization, cy-3 dNTPs were added to increase the signal intensity. (B) HBV mutations in patient sera identified using the dendron slide were compared with those identified by RFMP and sequencing.

RT, reverse transcriptase; ND, not determined; HBV, hepatitis B virus.

Moreover, the sensitivity and specificity of this technique are similar to or better than those of sequencing and RFMP. The merit of this technique is that patients' sera can be used directly without purification of viral DNA.

We confirmed that the selectivity and sensitivity of dendron slides are higher than those of conventional microarray slides (Figs 1-3). Microarray analysis using dendron slides can detect HBV DNA at a very low concentration ( 1 copy/ $\mu \mathrm{L})$, which might be helpful in detecting mutations in drug-resistant HBV when serum viremia is extremely low. Moreover, signal intensity was increased when cy3-dNTPs were added during hybridization (Figs 2 and 4). This signal enhancement may be due to the adhesion of cy3-dNTPs to DNA fragments. ${ }^{28}$ The added cy3dNTPs may polymerize on single-stranded regions of the HBV 
DNA fragments attached to the probe during hybridization. The remarkable sensitivity of dendron slides is related to the uniform and wide spacing of each probe, or mesospacing effect. In contrast to the high probe density on aldehyde slides, the sparse distribution on dendron slides makes it easier for the target PCR products to access the probes, which increases sensitivity and selectivity.

For diagnostic purposes, a simple process is preferable if its accuracy is guaranteed. When serum was directly used for microarray detection without viral DNA purification, signal quality and intensity were similar to those obtained using purified viral DNA (Fig 4A). Although some PCR inhibitors are known to be present in human serum, ${ }^{29}$ dendron slides were able to detect the correct signals from $17 \mathrm{CHB}$ serum samples, and the results were similar to those of RFMP and sequencing (Figs 4 and 6). Further studies employing large number of CHB samples will be needed to establish the diagnostic value of our method.

Among the tools used to detect HBV mutations, DNA microarray was developed most recently; however, it has not been widely used mainly due to the low specificity and high background signals. ${ }^{18}$ Nonetheless, DNA microarray has many advantages such as multigene detection on a single slide and low cost. For the detection of drug-resistant HBV mutants, the advantage of DNA microarray is that it can detect multiple mutations simultaneously. Moreover, by virtue of the mesospacing effect of dendron probes, dendron microarray could overcome certain drawbacks of conventional microarray including low signal intensity and specificity. ${ }^{23,24}$ RFMP technology is very sensitive and accurate, however, it requires access to a MALDITOF mass spectrometry which is expensive and needs trained operators. Although direct sequencing is a gold standard, it is expensive, time-consuming, labor-intensive, and difficult to detect the minor mutations and to adapt to high-throughput screening. According to our data, the dendron-modified DNA microarray does not require the purification of viral DNA and is easy to apply to high-throughput analysis, this is more costeffective assay compared with RFMP and sequencing.

The incidence of liver cancer is related to the amount of HBV DNA in patients. ${ }^{3}$ A recent report showed that low-level viremia is associated with a higher risk of HCC development compared to complete viral suppression. ${ }^{30}$ Thus, if a virus breakthrough occurs and is accompanied by an HBV drug-resistance mutation, it might be beneficial to provide rescue treatment as soon as possible in order to decrease viremia and subsequent hepatic inflammation. To implement this strategy, regular screening for drug-resistant HBV mutants is required. Therefore, there is a need for cost-effective, easy, and accurate methods for detection of such mutants. The prompt diagnosis of drug resistance leads to successful HBV treatment.

As many anti-HBV NAs have been developed over the last decades, the number of HBV drug-resistance mutations has also increased. The driving force of the generation of drug resis- tance mutations is the error-prone nature of $\mathrm{HBV}$ polymerase, with approximately 1 error per $10^{5}$ to $10^{7}$ bases polymerized. ${ }^{31}$ Therefore, drug-resistant strains appear easily and are selected under NA treatment. The well-known drug-resistance mutations include: rtL180M and rtM204I/V for LMV and clevudine; rtA181T/V and rtN236T for ADV; rtI169T, rtL180M, rtS202G/ I, rtM204I/V, rtS202G/I, and rtM250I/V for ETV; rtL80I/V, rtL180M, and rtM204I for telbivudine..$^{6,17,32,33} \mathrm{TDF}$, a recently developed antiviral NA drug, is a last-line-of-defense drug against HBV. Fortunately, there is no evidence of clinical resistance of HBV against TDF since it was introduced 6 years ago. ${ }^{34}$ However, there is still a possibility of the emergence of mutations that confer resistance to TDF if patients are treated for long time, because the efficacy of TDF is reduced by prior ADV treatment. ${ }^{35}$

Viral breakthroughs accompanied by drug resistance occur relatively frequently with the use of NA drugs. Thus, the development of rapid, cost-effective, and selective detection methods for HBV mutants is important and clinically necessary. In this regard, our study may contribute to the development of microarrays being capable of detecting drug-resistance mutations precisely and in a high-throughput manner.

\section{CONFLICTS OF INTEREST}

No potential conflict of interest relevant to this article was reported.

\section{ACKNOWLEDGEMENTS}

This study was supported by Konkuk University.

\section{REFERENCES}

1. Perz JF, Armstrong GL, Farrington LA, Hutin YJ, Bell BP. The contributions of hepatitis B virus and hepatitis C virus infections to cirrhosis and primary liver cancer worldwide. J Hepatol 2006;45:529-538.

2. Schiff ER. Prevention of mortality from hepatitis B and hepatitis C. Lancet 2006;368:896-897.

3. Chen CJ, Yang HI, Su J, et al. Risk of hepatocellular carcinoma across a biological gradient of serum hepatitis B virus DNA level. JAMA 2006;295:65-73.

4. Liaw YF, Sung JJ, Chow WC, et al. Lamivudine for patients with chronic hepatitis B and advanced liver disease. N Engl J Med 2004;351:1521-1531.

5. Hoofnagle JH, Doo E, Liang TJ, Fleischer R, Lok AS. Management of hepatitis B: summary of a clinical research workshop. Hepatology 2007;45:1056-1075.

6. Zoulim F, Locarnini S. Hepatitis B virus resistance to nucleos(t)ide analogues. Gastroenterology 2009;137:1593-1608.e2.

7. Yim HJ, Hwang SG. Options for the management of antiviral resistance during hepatitis B therapy: reflections on battles over a 
decade. Clin Mol Hepatol 2013;19:195-209.

8. Lee JH, Cho Y, Lee DH, et al. Prior exposure to lamivudine increases entecavir resistance risk in chronic hepatitis B Patients without detectable lamivudine resistance. Antimicrob Agents Chemother 2014;58:1730-1737.

9. Ou ZY, Liu N, Chen CJ, Cheng G, He YS. Rapid and accurate genotyping of YMDD motif variants in the hepatitis B virus genome by an improved reverse dot blot method. J Clin Microbiol 2005;43:5685-5689.

10. Whalley SA, Brown D, Teo CG, Dusheiko GM, Saunders NA. Monitoring the emergence of hepatitis $\mathrm{B}$ virus polymerase gene variants during lamivudine therapy using the LightCycler. J Clin Microbiol 2001;39:1456-1459.

11. Libbrecht E, Doutreloigne J, Van De Velde H, et al. Evolution of primary and compensatory lamivudine resistance mutations in chronic hepatitis B virus-infected patients during long-term lamivudine treatment, assessed by a line probe assay. J Clin Microbiol 2007;45:3935-3941.

12. Lindström A, Odeberg J, Albert J. Pyrosequencing for detection of lamivudine-resistant hepatitis B virus. J Clin Microbiol 2004;42:4788-4795.

13. Kirishima T, Okanoue T, Daimon Y, et al. Detection of YMDD mutant using a novel sensitive method in chronic liver disease type B patients before and during lamivudine treatment. J Hepatol 2002;37:259-265.

14. Jang H, Cho M, Heo J, et al. Oligonucleotide chip for detection of Lamivudine-resistant hepatitis B virus. J Clin Microbiol 2004;42:4181-4188.

15. Hong SP, Kim NK, Hwang SG, et al. Detection of hepatitis B virus YMDD variants using mass spectrometric analysis of oligonucleotide fragments. J Hepatol 2004;40:837-844.

16. Lee CH, Kim SO, Byun KS, et al. Predominance of hepatitis B virus YMDD mutants is prognostic of viral DNA breakthrough. Gastroenterology 2006;130:1144-1152.

17. Kim JH, Park YK, Park ES, Kim KH. Molecular diagnosis and treatment of drug-resistant hepatitis B virus. World J Gastroenterol 2014;20:5708-5720.

18. Heo J, Cho M, Kim HH, et al. Detection of YMDD motif mutants by oligonucleotide chips in lamivudine-untreated patients with chronic hepatitis B virus infection. J Korean Med Sci 2004;19:541546.

19. Vernet G, Tran N. The DNA-Chip technology as a new molecular tool for the detection of HBV mutants. J Clin Virol 2005;34 Suppl 1:S49-S53.

20. Pas SD, Tran N, de Man RA, Burghoorn-Maas C, Vernet G, Niesters HG. Comparison of reverse hybridization, microarray, and sequence analysis for genotyping hepatitis B virus. J Clin Microbiol 2008;46:1268-1273.

21. Kwon SH, Hong BJ, Park HY, Knoll W, Park JW. DNA-DNA inter- action on dendron-functionalized sol-gel silica films followed with surface plasmon fluorescence spectroscopy. J Colloid Interface Sci 2007;308:325-331.

22. Hong BJ, Oh SJ, Youn TO, Kwon SH, Park JW. Nanoscale-controlled spacing provides DNA microarrays with the SNP discrimination efficiency in solution phase. Langmuir 2005;21:4257-4261.

23. Hong BJ, Sunkara V, Park JW. DNA microarrays on nanoscalecontrolled surface. Nucleic Acids Res 2005;33:e106.

24. Oh SJ, Ju J, Kim BC, et al. DNA microarrays on a dendron-modified surface improve significantly the detection of single nucleotide variations in the p53 gene. Nucleic Acids Res 2005;33:e90.

25. Lim KH, Park ES, Kim DH, et al. Suppression of interferon-mediated anti-HBV response by single CpG methylation in the 5'-UTR of TRIM22. Gut 2018;67:166-178.

26. Park YK, Park ES, Kim DH, et al. Cleaved c-FLIP mediates the antiviral effect of TNF-alpha against hepatitis B virus by dysregulating hepatocyte nuclear factors. J Hepatol 2016;64:268-277.

27. Kim KT, Na CH, Yun YM, Hwang TS, Kim SN, Chae CB. Dramatic increase in signal by integration of polymerase chain reaction and hybridization on surface of DNA microarray. Anal Biochem 2010;396:139-145.

28. Tjong V, Yu H, Hucknall A, Rangarajan S, Chilkoti A. Amplified on-chip fluorescence detection of DNA hybridization by surfaceinitiated enzymatic polymerization. Anal Chem 2011;83:51535159.

29. Klein A, Barsuk R, Dagan S, Nusbaum O, Shouval D, Galun E. Comparison of methods for extraction of nucleic acid from hemolytic serum for PCR amplification of hepatitis B virus DNA sequences. J Clin Microbiol 1997;35:1897-1899.

30. Kim JH, Sinn DH, Kang W, et al. Low-level viremia and the increased risk of hepatocellular carcinoma in patients receiving entecavir treatment. Hepatology 2017;66:335-343.

31. Caligiuri P, Cerruti R, Icardi G, Bruzzone B. Overview of hepatitis $B$ virus mutations and their implications in the management of infection. World J Gastroenterol 2016;22:145-154.

32. Kim KH, Kim ND, Seong BL. Discovery and development of antiHBV agents and their resistance. Molecules 2010;15:5878-5908.

33. Ahn SH, Park YK, Park ES, et al. The impact of the hepatitis B virus polymerase rtA181T mutation on replication and drug resistance is potentially affected by overlapping changes in surface gene. J Virol 2014;88:6805-6818.

34. Corsa AC, Liu Y, Flaherty JF, et al. No resistance to tenofovir disoproxil fumarate through 96 weeks of treatment in patients with lamivudine-resistant chronic hepatitis B. Clin Gastroenterol Hepatol 2014;12:2106-2112.e1.

35. Chung GE, Cho EJ, Lee JH, et al. Tenofovir has inferior efficacy in adefovir-experienced chronic hepatitis B patients compared to nucleos(t)ide-naïve patients. Clin Mol Hepatol 2017;23:66-73. 